\title{
Recombinant RANTES
}

National Cancer Institute

\section{Source}

National Cancer Institute. Recombinant RANTES. NCI Thesaurus. Code C1211.

A member of the IL-8 superfamily of cytokines and released from platelets and activated T-cells, RANTES is a selective chemoattractant for eosinophils, monocytes, and Tlymphocytes. RANTES is one of the ligands for chemokine receptor CCR5. RANTES expression in peripheral lymphocytes increases exponentially following mitogenic or antigenic stimulation. RANTES is one of the major HIV-suppressive factors produced by CD8-positive T-cells. 